\title{
Endocarditis prophylaxis: from experimental models to human recommendation
}

\author{
M. Blatter aNd P. Francioli
}

\author{
Division des Maladies Infectieuses et Division Autonome de Médecine Préventive Hospitalière, Centre Hospitalier \\ Universitaire Vaudois, Lausanne, Switzerland
}

KEY WORDS: Endocarditis, prophylaxis, antibiotics.

\begin{abstract}
Animal models of endocarditis have helped understanding of the mode of action of antibiotics in prophylaxis. During bacteraemia, some microorganisms will adhere to damaged cardiac valves. The proportion of bacteria that will adhere depends largely on intrinsic properties of the strain. In the absence of antibiotics, the microorganisms will either be eliminated by local host defence mechanisms if the inoculum is low enough or will begin to grow approximately $2 \mathrm{~h}$ after the development of bacteraemia and endocarditis. In the presence of antibiotics, the growth of adherent bacteria is inhibited and local host defences have longer to eliminate the bacteria. In cases where the number of adherent bacteria is relatively low, a short period in inhibitory antibiotic activity (6 hours) is sufficient to eliminate the bacteria and lead to successful prophylaxis. If the number of bacteria is high, a longer duration of the inhibitory effect is necessary. These experimental data provide a rationale for practical recommendations for the prophylaxis of endocarditis in humans.
\end{abstract}

\section{Introduction}

Recommendations for the antibiotic prophylaxis of endocarditis are updated periodically in order to fit new data and practices. Unfortunately, there is no prospective clinical trial on the efficacy of prophylactic antibiotics in endocarditis, and it is unlikely that such a study will ever be performed, both for ethical and practical reasons. Two case-control studies have shown a prophylactic efficacy of antibiotics of $49 \%$ and $91 \%$, in patients with native valves $^{[1,2]}$. A French study showed no protective efficacy ${ }^{[3]}$. In another study of patients with prosthetic valves and a multiple-dose prophylactic regimen, six cases of endocarditis were recorded following 390 invasive procedures performed without antibiotic prophylaxis whereas none were recorded among 285 procedures performed with antibiotic prophylaxis, a difference which was statistically significant ${ }^{[4]}$. These divergent results may partially be explained by the characteristics of the antibiotic regimens used. At present, most European countries recommend a single oral dose of $3 \mathrm{~g}$ of amoxicillin to be taken 1 hour before a procedure. In contrast, the American Heart Association recommend a second dose of $1.5 \mathrm{~g}$ of amoxicillin, to be given $6 \mathrm{~h}$ after the first ${ }^{|5|}$. Some countries, including Switzerland, differentiate between moderateand high-risk patients (prosthetic valves, prior endocarditis) and recommend multiple doses of antibiotics only for the high-risk patients ${ }^{(\theta)}$. There are no human data to support any particular one of these various approaches. However, experimental models provide helpful information for practical recommendations.

Correspondence: P. Francioli, MD, Division autonome de médecine preventive hospitalière, Centre Hospitalier Universitaire Vaudois, 1011 Lausanne, Switzerland.

\section{Role of the inoculum size}

In their early experiments on rabbits, Durack and his colleagues injected i.v. $10^{8}$ colony-forming units (cfu) of viridans streptococci and enterococci, an inoculum size that produced endocarditis in $100 \%$ of the control animals ${ }^{[7]}$. In fact, this number of bacteria was probably 10 to 100 times higher than the lowest infecting dose necessary to produce endocarditis in $90 \%$ of the rabbits $\left(\mathrm{ID}_{90}\right)$. Various regimens of prophylactic antibiotics were tested under these stringent experimental conditions and as results suggested that prolonged administration of high doses of antibiotics were necessary for successful prophylaxis, multiple dose regimens were recommended in humans ${ }^{[8]}$. These experiments were criticized because of the high inoculum used, which was thought to be irrelevant to the human situation. In experiments performed more recently in the rat model of endocarditis, the efficacy of the prophylactic antibiotics was found to be dependent on the inoculum size used for the bacterial challenge: protection was excellent with single dose of antibiotics for inocula not higher than the $\mathrm{ID}_{90}$ but disappeared with higher inocula ${ }^{[9]}$. As observed by Durack and colleagues, protection was reestablished in the multiple doses of antibiotics ${ }^{[10]}$. These results were thought to provide a good rationale for single dose prophylaxis regimens, as very high inocula were unlikely to apply to human situations. The magnitude of bacteraemia observed in humans after certain procedures such as teeth extraction is generally of the order of $10^{1}$ to

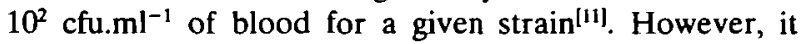
should be stressed that the number of bacteria injected i.v. into animals does not represent the number of bacteria that circulate, due to both a passive haemodilution and an active clearance mechanism by the reticulo-endothelial system $^{[12]}$. In addition, studies on endocarditis in rats with periodontitis and after teeth extraction have failed to 
demonstrate a relationship between the number of a given streptococcal species circulating immediately after teeth extraction and the likelihood of these streptococci subsequently producing endocarditis ${ }^{[13]}$. Indeed, some streptococcal strains found in the blood at barely detectable levels consistently produced endocarditis, while other viridans streptococci found in much higher numbers in the blood only rarely infected the valves. In these experiments, the parameter that best predicted the likelihood of a strain producing endocarditis was the in vitro adherence of the strain to platelet-fibrin matrices ${ }^{[13]}$. Thus, the determination of the magnitude of the bacteraemia alone may not provide reliable information on the risk of subsequent development of bacterial endocarditis after certain procedures. Finally, quantitative blood cultures obtained from rats after the i.v. injection of $10^{4}$ cfu of either Streptococcus intermedius or $S$. faecalis (corresponding approximately to the $\mathrm{ID}_{5_{0}}$ and $\mathrm{ID}_{90}$ of the two microorganisms, respectively) showed that the magnitude of the bacteraemia was in the range of $10^{2}$ cfu. $\mathrm{ml}^{-1}$ at $5 \mathrm{~min}^{[12]}$. These data are similar to those reported in humans after certain invasive procedures such as teeth extraction ${ }^{[11]}$. Occasionally, figures of $10^{5}$ cfu. $\mathrm{ml}^{-1}$ have been documented. The relationship between the magnitude of the bacteraemia and the subsequent risk of developing endocarditis is unknown in humans. It may well be that the very few patients who will develop endocarditis after procedures are precisely those with the highest number of circulating bacteria or those with bacteria with high adherence properties. Thus, some countries have elected to recommend a high dose of amoxicillin 1 hour before the procedure followed by one or several additional doses a few hours later ${ }^{[5,6]}$.

\section{Inhibitory and bactericidal activity of the antibiotics}

It is still believed by some authors that the protective effect of the antibiotics in the prophylaxis of endocarditis is due to the bactericidal action of the antibiotic, and that high serum levels at the time of bacteraemia should provide optimal bactericidal activity and protection. There are several lines of evidence which suggest that the bactericidal activity is not the main mechanism of action of antibiotics in endocarditis prophylaxis. First, when carefully tested in vitro, many strains of viridans streptococci as well as enterococci are tolerant to the bactericidal action of amoxicillin $^{[14]}$. Nevertheless, amoxicillin is effective in preventing experimental endocarditis due to these strains ${ }^{[9.12]}$. Second, antibiotics also provide protection when administered after bacterial challenge, clearly showing that the prophylactic effect is not due to a bactericidal activity on the circulating bacteria $^{[15]}$. Thus, other mechanisms of action have to be considered and experiments suggest that antibiotics operate in inhibiting of the growth of the bacteria stuck to the vegetations, thereby allowing other elimination mechanisms to operate ${ }^{[12]}$. This concept is based on the following observations: after bacterial challenge, a small proportion of the circulating bacteria will adhere to the vegetations within the first few minutes, but they will tend to disappear spontaneously from the valves even in the absence of antibiotics ${ }^{[12]}$. However, if too many bacteria have adhered their spontaneous elimination will be overcome by the growth of the bacteria, and endocarditis will occur. If growth can be retarded by an inhibitory concentration of antibiotics, elimination will continue and endocarditis may be prevented if all adherent bacteria have been cleared before bacterial growth resumes. The higher the inoculum (i.e. the higher the amount of adherent bacteria), the more prolonged the inhibitory concentration of antibiotics to prevent endocarditis should be ${ }^{[12]}$.

This concept was confirmed in recent experiments which compared the efficacy of sustained low versus short high blood levels of amoxicillin in the prevention of streptococcal endocarditis in the rat. Results showed that amoxicillin administered as a continuous infusion over $12 \mathrm{~h}$ was significantly more effective than an i.v. bolus of the same amount of the antibiotic (antibiotic no longer detectable in the serum at $6 \mathrm{~h}$ ). Moreover, a low dose of amoxicillin was equally as active as a high dose when administered continuously over $12 \mathrm{~h}^{[16]}$.

Thus, bacterial growth inhibition mediated by the presence of inhibitory concentration of antibiotics over several hours could be an important preventive mechanism of the antibiotics in allowing bacterial clearance of resting organisms at the surface of the vegetations by as yet undefined host defence factors. Platelets have been shown to contain bactericidal substances. Since they are major constituents of the vegetations, they may play an important adjuvant role in killing the bacteria present at the surface of a vegetation $^{[17]}$.

\section{From animals to humans}

The arguments for the single dose regimen in humans have been the following: an oral dose of $3 \mathrm{~g}$ of amoxicillin taken $30 \mathrm{~min}$ before a procedure produces serum levels at the time of the procedure which are much higher than the minimal inhibitory and bactericidal concentrations of most potential pathogens circulating in the blood. In addition, serum levels are detectable for at least 6 hours. Such an oral dose is easy to administer, well tolerated and should ensure optimal compliance. Experimentally, in conditions mimicking single dose amoxicillin prophylaxis, protection was afforded for inocula infecting $90 \%$ of control animals; it is therefore likely to provide a good margin of safety in humans since the number of circulating bacteria during an invasive procedure is usually very low. This approach has been chosen by The Netherlands, U.K. and France.

In contrast, some authorities recommend that patients at risk for endocarditis take a high dose of amoxicillin before an invasive procedure as well as one or several additional doses in order to cover the possibility that the heart valves may have been seeded by a rather high number of bacteria. The experimental data described above suggest that such an approach is likely to provide a higher margin of safety. The American Heart Association recommends one additional dose of amoxicillin for all patients. The present Swiss and German recommendations distinguish between patients at low and high risk for endocarditis. For those at high risk (prosthetic valves, prior endocarditis), seven additional doses of amoxicillin are presently recommended ${ }^{[6]}$. 


\section{References}

[1] van der Meer JTM, van Wijk W, Thompson J, Vandenbroucke JP, Valkenburg HA, Michel MF. Efficacy of antibiotic prophylaxis for prevention of native-valve endocarditis. Lancet 1992; 339: 135-9.

[2] Imperiale TF, Horwitz RI. Does prophylaxis prevent postdental infective endocarditis? A controlled evaluation of protective efficacy. Am J Med 1990; 88: 131-6.

[3] Hoen B, Lacassin F, Briancon $S$ et al. Gestes à risque d'endocardite infectieuse. Une enquête cas-témoins. Méd Mal Infect 1992; 22(spécial): 1010-22.

[4] Horstkotte D, Friedrichs W, Pippert H, Bricks W, Loogen F. Nutzen der Endokarditisprophylaxe bei Patienten mit prothetischen Herzklappen. Z Kardiol 1986; 75: 8-11.

[5] Dajani AS, Bisno AL, Chung KJ, Durack DT. Prevention of Bacterial Endocarditis. Recommendations by the American Heart Association. J Am Med Assoc 1990; 264; 2919-22.

[6] Malinverni R, Francioli P, Gerber A et al. Prophylaxe der Bakteriellen Endokarditis. Empfehlungen der Schweizerischen Arbeitsgruppe für Endokarditisprophylaxe. Schweiz med Wschr 1984; 37: 1246-52.

[7] Durack DT, Petersdorf RG. Chemotherapy of experimental streptococcal endocarditis: 1 . Comparison of commonly recommended prophylactic regimens. J Clin Invest 1973; 52: 592-8.

[8] American Heart Association Committee Report. Prevention of bacterial endocarditis. Circulation 1922; 56: 139A-143A.

[9] Glauser MP, Bernard JP, Moreillon P, Francioli P. Successful single-dose amoxicillin prophylaxis against experimental endocarditis: evidence for two mechanisms of protection. $J$ Infect Dis 1983; 147: 568-75.

[10] Malinverni R, Francioli P, Glauser MP. Comparison of single and multiple doses of prophylactic antibiotics in experimental streptococcal endocarditis. Circulation 1987; 76: 376-82.

[11] Everett ED, Hirschmann JV. Transient bacteremia and endocarditis prophylaxis: A review. Medicine 1977; 56: 61-77.

[12] Moreillon P, Francioli P, Overholser CD, Meylan P, Glauser MP. Mechanisms of successful amoxicillin prophylaxis of experimental endocarditis due to Streptococcus intermedius. $\mathrm{J}$ Inf Dis 1986; 154: 801-7.

[13] Overholser CD, Moreillon P, Glauser MP. Experimental bacterial endocarditis after dental extractions in rats with peritonitis. J Inf Dis 1987; 155: 107-12.

[14] Meylan PR, Francioli P, Glauser MP. Discrepancies between $\mathrm{MBC}$ and actual killing of viridans group streptococci by cellwall-active antibiotics. Antimicrob Agents Chemother 1986; 29: 418-23.

[15] Bemey P, Francioli P. Successful prophylaxis of experimental streptococcal endocarditis with single dose amoxicillin administered after bacterial challenge. J Inf Dis 1990; 161: $281-5$.

[16] Fluckiger U, Glauser MP, Francioli P. Comparison of the efficacy of sustained low with short high blood levels of amoxicillin the prevention of streptococcal endocarditis in rats (abstract). ICAAC 1991.

[17] Sullam PM, Frank U, Yeaman MR, Tauber MG, Bayer AS, Chambers $\mathrm{H}$. Effect of thrombocytopenia on the early course of streptococcal endocarditis. J Inf Dis 1993; 168: 